\title{
Stability and Andronov-Hopf Bifurcation of a System with Three Time Delays
}

\author{
Svetoslav Nikolov ${ }^{1,2}$ \\ ${ }^{1}$ Department of Biomechanics, Institute of Mechanics-BAS, Acad. G. Bonchev Street, Block 4, 1113 Sofia, Bulgaria \\ ${ }^{2}$ Department of Mechanics, University of Transport, Geo Milev Street, 158, 1574 Sofia, Bulgaria
}

Correspondence should be addressed to Svetoslav Nikolov; s.nikolov@imbm.bas.bg

Received 7 November 2012; Revised 25 January 2013; Accepted 26 January 2013

Academic Editor: Zindoga Mukandavire

Copyright (C) 2013 Svetoslav Nikolov. This is an open access article distributed under the Creative Commons Attribution License, which permits unrestricted use, distribution, and reproduction in any medium, provided the original work is properly cited.

\begin{abstract}
A general system of three autonomous ordinary differential equations with three discrete time delays is considered. With respect to the delays, we investigate the local stability of equilibria by analyzing the corresponding characteristic equation. Using the Hopf bifurcation theorem, we predict the occurrence of a limit cycle bifurcation for the time delay parameters. Thus, some new mathematical results are obtained. Finally, the above mentioned criteria are applied to a system modelling miRNA regulation.
\end{abstract}

\section{Introduction}

The future status of many systems arising from engineering, physics, mechanics, biochemistry, or systems in biology is determined not only by their current behavior but is also by their history. Such phenomena are called delay or genetic effects. Time lags in continuous systems can produce complex dynamics and instabilities. In the recent years, many mathematical models that have appeared in the literature involve either a single discrete delay to investigate the role of phosphorylation, negative or positive feedback regulation of transcription factors [1-4], and gene expression multistability [5]. Several papers [6-13] among others consider systems with two or three discrete delays. When there is more than one delay in the equations, the local theory for stability is not fully complete.

A dynamical system which is not finite dimensional is called an infinite dimensional dynamical system. A class of infinite dimensional system can be determined by functional differential equations of the retarded type. To introduce such equations, we let $C_{r}$ denote the set $C\left[[-r, 0], R^{n}\right]$ with the norm defined by $\|\phi\|=\max \{|\phi(t):-r \leq t \leq 0|\}$ where $\|\cdot\|$ denotes a norm (e.g., the Euclidean norm). Given a function $x(\cdot)$ defined on $-r \leq t \leq 0$, let $x_{t}$ be the function determined by $x_{t}(s)=x(t+s)$ for $-r \leq s \leq 0$. A retarded functional differential equation (with delay $r$ ) is an equation of the form

$$
\dot{x}(t)=F\left(t, x_{t}\right),
$$

where $F \in C\left[\Omega, R^{n}\right]$ and $\Omega$ is an open set in $\left(R \times C_{r}\right)$. The notations of the dynamical system are determined by varying $\left(t_{0}, x_{t_{0}}=\psi\right)$ over some appropriate subset of $\Omega$. As far as we know, the first statement similar to the Hopf theorem (concerning the bifurcation of periodic solutions from a singular point of an ordinary differential equation) [14] for retarded functional differential equations was given in [15].

In this paper, we consider stability and bifurcation behavior of a three-dimensional system of autonomous ordinary differential delay equations of the form

$$
\begin{aligned}
x^{\prime}(t) & =f(x(t), x(t-\zeta), y(t), y(t-\xi), z(t), z(t-\eta)), \\
y^{\prime}(t) & =g(x(t), x(t-\zeta), y(t), y(t-\xi), z(t), z(t-\eta)), \\
z^{\prime}(t) & =h(x(t), x(t-\zeta), y(t), y(t-\xi), z(t), z(t-\eta)),
\end{aligned}
$$

where $\left({ }^{\prime}=d / d t\right) ; f, g, h: R \times R \times R \times R \times R \times R \rightarrow R$, are such that solutions to initial value problems exist and are continuable.

\section{Derivation of the Characteristic Equation}

We assume that $f, g$, and $h$ have continuous first partial derivatives with respect to their arguments and that there exists unique $\bar{x}>0, \bar{y}>0$, and $\bar{z}>0$, such that $f(\bar{x}, \bar{x}, \bar{y}, \bar{y}, \bar{z}, \bar{z})=g(\bar{x}, \bar{x}, \bar{y}, \bar{y}, \bar{z}, \bar{z})=h(\bar{x}, \bar{x}, \bar{y}, \bar{y}, \bar{z}, \bar{z})=0$. 
We utilize the following notation: $f_{i}, i=1, \ldots, 6$ that represents the partial derivative of $f$ with respect to its $i$ th argument evaluated $\bar{E}(\bar{x}, \bar{x}, \bar{y}, \bar{y}, \bar{z}, \bar{z})$. A similar meaning is given to $g_{j}, j=1, \ldots, 6$, and $h_{k}, k=1, \ldots, 6$. Hence, for example, $f_{2}=(\partial f(\bar{x}, \bar{x}, \bar{y}, \bar{y}, \bar{z}, \bar{z}) / \partial x(t-\zeta))$ and $h_{2}=$ $(\partial h(\bar{x}, \bar{x}, \bar{y}, \bar{y}, \bar{z}, \bar{z}) / \partial x(t-\zeta))$. The variational system with respect to $\bar{E}$ is

$$
\begin{aligned}
\left(\begin{array}{l}
u(t) \\
v(t) \\
\rho(t)
\end{array}\right)^{\prime}= & \left(\begin{array}{lll}
f_{1} & f_{3} & f_{5} \\
g_{1} & g_{3} & g_{5} \\
h_{1} & h_{3} & h_{5}
\end{array}\right)\left(\begin{array}{l}
u(t) \\
v(t) \\
\rho(t)
\end{array}\right) \\
& +\left(\begin{array}{lll}
f_{2} & f_{4} & f_{6} \\
g_{2} & g_{4} & g_{6} \\
h_{2} & h_{4} & h_{6}
\end{array}\right)\left(\begin{array}{c}
u(t-\zeta) \\
v(t-\xi) \\
\rho(t-\eta)
\end{array}\right) .
\end{aligned}
$$

We seek a solution of (3) of the form $u(t)=c_{1} e^{\chi t}, v(t)=c_{2} e^{\chi t}$ and $\rho(t)=c_{3} \ell^{X t}$. For nontrivial solutions, this leads to the following characteristic equation in $\chi$ :

$$
\begin{aligned}
\chi^{3} & +a_{1} \chi^{2}+a_{2} \chi+a_{3}+\ell^{-\chi \zeta}\left(a_{4} \chi^{2}+a_{7} \chi+a_{13}\right) \\
& +\ell^{-\chi \xi}\left(a_{5} \chi^{2}+a_{8} \chi+a_{14}\right)+\ell^{-\chi \eta}\left(a_{6} \chi^{2}+a_{9} \chi+a_{15}\right) \\
& +\ell^{-\chi(\zeta+\xi)}\left(a_{10} \chi+a_{16}\right)+\ell^{-\chi(\zeta+\eta)}\left(a_{11} \chi+a_{17}\right) \\
& +e^{-\chi(\xi+\eta)}\left(a_{12} \chi+a_{18}\right)+a_{19} \ell^{-\chi(\zeta+\xi+\eta)}=0,
\end{aligned}
$$

where

$$
\begin{aligned}
a_{1}= & -\left(f_{1}+g_{3}+h_{5}\right), \\
a_{2}= & f_{1}\left(g_{3}+h_{5}\right)-f_{3} g_{1}-f_{5} h_{1}+g_{3} h_{5}-g_{5} h_{3}, \\
a_{3}= & f_{1}\left(g_{5} h_{3}-g_{3} h_{5}\right)+f_{3}\left(g_{1} h_{5}-g_{5} h_{1}\right) \\
& +f_{5}\left(g_{3} h_{1}-g_{1} h_{3}\right), \\
a_{4}= & -f_{2}, \quad a_{5}=-g_{4}, \quad a_{6}=-h_{6}, \\
a_{7}= & f_{2}\left(g_{3}+h_{5}\right)-f_{3} g_{2}-f_{5} h_{2}, \\
a_{8}= & g_{4}\left(f_{1}+h_{5}\right)-f_{4} g_{1}-g_{5} h_{4}, \\
a_{9}= & h_{6}\left(f_{1}+g_{3}\right)-f_{6} h_{1}-g_{6} h_{3}, \\
a_{10}= & f_{2} g_{4}-f_{4} g_{2}, \quad a_{11}=f_{2} h_{6}-f_{6} h_{2}, \\
a_{12}= & g_{4} h_{6}-g_{6} h_{4}, \\
a_{13}= & f_{2}\left(g_{5} h_{3}-g_{3} h_{5}\right)+f_{3}\left(g_{2} h_{5}-g_{5} h_{2}\right) \\
& +f_{5}\left(g_{3} h_{2}-g_{2} h_{3}\right), \\
a_{14}= & f_{1}\left(g_{5} h_{4}-g_{4} h_{5}\right)+f_{4}\left(g_{1} h_{5}-g_{5} h_{1}\right) \\
& +f_{5}\left(g_{4} h_{1}-g_{1} h_{4}\right), \\
a_{15}= & f_{1}\left(g_{6} h_{3}-g_{3} h_{6}\right)+f_{3}\left(g_{1} h_{6}-g_{6} h_{1}\right) \\
& +f_{6}\left(g_{3} h_{1}-g_{1} h_{3}\right), \\
&
\end{aligned}
$$

$$
\begin{aligned}
a_{16}= & f_{2}\left(g_{5} h_{4}-g_{4} h_{5}\right)+f_{4}\left(g_{2} h_{5}-g_{5} h_{2}\right) \\
& +f_{5}\left(g_{4} h_{2}-g_{2} h_{4}\right), \\
a_{17}= & f_{2}\left(g_{6} h_{3}-g_{3} h_{6}\right)+f_{3}\left(g_{2} h_{6}-g_{6} h_{2}\right) \\
& +f_{6}\left(g_{3} h_{2}-g_{2} h_{3}\right), \\
a_{18}= & f_{1}\left(g_{6} h_{4}-g_{4} h_{6}\right)+f_{4}\left(g_{1} h_{6}-g_{6} h_{1}\right) \\
& +f_{6}\left(g_{4} h_{1}-g_{1} h_{4}\right), \\
a_{19}= & f_{2}\left(g_{6} h_{4}-g_{4} h_{6}\right)+f_{4}\left(g_{2} h_{6}-g_{6} h_{2}\right) \\
& +f_{6}\left(g_{4} h_{2}-g_{2} h_{4}\right) .
\end{aligned}
$$

Remark 1. We note that $\chi=0$ is a root of (4) if and only if $a_{3}=a_{13}=a_{14}=a_{15}=a_{16}=a_{17}=a_{18}=a_{19}=0$.

It is well known that the stability of the equilibrium state $\bar{E}$ depends on the sign of the real parts of the roots of (4). If $\chi=$ $m+i n(m, n \in R)$ satisfies (4), then $m$ and $n$ are real solutions; that is, we rewrite (4) in terms of its real and imaginary parts as

$$
\begin{gathered}
m^{3}-3 m n^{2}+a_{1}\left(m^{2}-n^{2}\right)+a_{2} m+a_{3} \\
+\ell^{-m \zeta}\left[a_{4}\left(m^{2}-n^{2}\right) \cos n \zeta+2 a_{4} m n \sin n \zeta\right. \\
\left.+a_{7}(m \cos n \zeta+n \sin n \zeta)+a_{13} \cos n \zeta\right] \\
+\ell^{-m \xi}\left[a_{5}\left(m^{2}-n^{2}\right) \cos n \xi+2 a_{5} m n \sin n \xi\right. \\
\left.+a_{8}(m \cos n \xi+n \sin n \xi)+a_{14} \cos n \xi\right] \\
+\ell^{-m \eta}\left[a_{6}\left(m^{2}-n^{2}\right) \cos n \eta+2 a_{6} m n \sin n \eta\right. \\
\left.+a_{9}(m \cos n \eta+n \sin n \eta)+a_{15} \cos n \eta\right] \\
+\ell^{-m(\zeta+\xi)}\left[a_{10}(m \cos n(\zeta+\xi)+n \sin n(\zeta+\xi))\right. \\
\left.+a_{16} \cos n(\zeta+\xi)\right] \\
+\ell^{-m(\zeta+\eta)}\left[a_{11}(m \cos n(\zeta+\eta)+n \sin n(\zeta+\eta))\right. \\
\left.+a_{17} \cos n(\zeta+\eta)\right] \\
+\ell^{-m(\xi+\eta)}\left[a_{12}(m \cos n(\xi+\eta)+n \sin n(\xi+\eta))\right. \\
\left.+a_{18} \cos n(\xi+\eta)\right] \\
+a_{19} \ell^{-m(\zeta+\xi+\eta)} \cos n(\zeta+\xi+\eta)=0 \\
3 m^{2} n-n^{3}+2 a_{1} m n+a_{2} n \\
+\ell^{-m \zeta}\left[2 a_{4} m n \cos n \zeta-a_{4}\left(m^{2}-n^{2}\right) \sin n \zeta\right. \\
\left.\cos n \zeta-m \sin n \zeta)-a_{13} \sin n \zeta\right]
\end{gathered}
$$




$$
\begin{gathered}
+\ell^{-m \xi}\left[2 a_{5} m n \cos n \xi-a_{5}\left(m^{2}-n^{2}\right) \sin n \xi\right. \\
\left.+a_{8}(n \cos n \xi-m \sin n \xi)-a_{14} \sin n \xi\right] \\
+\ell^{-m \eta}\left[2 a_{6} m n \cos n \eta-a_{6}\left(m^{2}-n^{2}\right) \sin n \eta\right. \\
\left.+a_{9}(n \cos n \eta-m \sin n \eta)-a_{15} \sin n \eta\right] \\
+\ell^{-m(\zeta+\xi)}\left[a_{10}(n \cos n(\zeta+\xi)-m \sin n(\zeta+\xi))\right. \\
\left.-a_{16} \sin n(\zeta+\xi)\right] \\
+\ell^{-m(\zeta+\eta)}\left[a_{11}(n \cos n(\zeta+\eta)-m \sin n(\zeta+\eta))\right. \\
\left.-a_{17} \sin n(\zeta+\eta)\right] \\
+\ell^{-m(\xi+\eta)}\left[a_{12}(n \cos (\xi+\eta)-m \sin n(\xi+\eta))\right. \\
\left.-a_{18} \sin n(\xi+\eta)\right] \\
-a_{19} \ell^{-m(\zeta+\xi+\eta)} \sin n(\zeta+\xi+\eta)=0 .
\end{gathered}
$$

In the absence of delays $(\zeta=\xi=\eta=0), \bar{E}$ is locally asymptotically stable if

$$
\begin{gathered}
p=a_{1}+a_{4}+a_{5}+a_{6}>0, \\
q=a_{2}+a_{7}+a_{8}+a_{9}+a_{10}+a_{11}+a_{12}>0, \\
r=a_{3}+a_{13}+a_{14}+a_{15}+a_{16}+a_{17}+a_{18}+a_{19}>0, \\
R=p q-r>0 .
\end{gathered}
$$

Because of the presence of three different discrete delays in (2), the analysis of the sign of the real parts of the eigenvalues is very complicated, and a direct approach cannot be considered. Thus, in our analysis we will use a method consisting of determining the stability of the steady state when firstly two delays are equal to zero, and when secondly one delay is equal to zero. Previously, this approach is used for system with two delays $[9,12,13,16-18]$.

\section{The Case $\zeta=\xi=0$ and $\eta>0$}

Setting $\zeta=\xi=0$ in (4), the system (6) becomes

$$
\begin{aligned}
m^{3}-3 m n^{2} & +K_{1}\left(m^{2}-n^{2}\right)+K_{2} m+K_{3} \\
+\ell^{-m \eta} & {\left[T_{1}(m \cos n \eta+n \sin n \eta)\right.} \\
& \left.+\cos n \eta\left(T_{2}+a_{6}\left(m^{2}-n^{2}\right)\right)+2 a_{6} m n \sin n \eta\right]=0 \\
3 m^{2} n-n^{3}+ & 2 K_{4} m n+K_{2} n \\
+\ell^{-m \eta} & {\left[T_{1}(n \cos n \eta-m \sin n \eta)\right.} \\
& \left.-\sin n \eta\left(T_{2}+a_{6}\left(m^{2}-n^{2}\right)\right)\right]=0,
\end{aligned}
$$

where

$$
\begin{gathered}
K_{1}=a_{1}+a_{4}+a_{5}, \quad K_{2}=a_{2}+a_{7}+a_{8}+a_{10}, \\
K_{3}=a_{3}+a_{13}+a_{14}+a_{16}, \quad K_{4}=a_{1}+a_{4}+a_{5}+a_{6}, \\
T_{1}=a_{9}+a_{11}+a_{12}, \quad T_{2}=a_{15}+a_{17}+a_{18}+a_{19} .
\end{gathered}
$$

To find the first bifurcation point, we look for purely imaginary roots $\chi= \pm i n, n \in R$ of (4) (when $\zeta=\xi=0$ ); that is, we set $m=0$. Then, the above two equations are reduced to

$$
\begin{gathered}
-K_{1} n^{2}+K_{3}=-n T_{1} \sin n \eta-\left(T_{2}-a_{6} n^{2}\right) \cos n \eta \\
-n^{3}+K_{2} n=-n T_{1} \cos n \eta+\left(T_{2}-a_{6} n^{2}\right) \sin n \eta
\end{gathered}
$$

or another one

$$
\begin{aligned}
& \cos n \eta=\frac{\left(n^{2}-K_{2}\right) n^{2} T_{1}+\left(T_{2}-a_{6} n^{2}\right)\left(K_{1} n^{2}-K_{3}\right)}{n^{2} T_{1}^{2}+\left(T_{2}-a_{6} n^{2}\right)^{2}}, \\
& \sin n \eta=\frac{\left(K_{1} n^{2}-K_{3}\right) n T_{1}-n\left(T_{2}-a_{6} n^{2}\right)\left(n^{2}-K_{2}\right)}{n^{2} T_{1}^{2}+\left(T_{2}-a_{6} n^{2}\right)^{2}} .
\end{aligned}
$$

We note here that it is not possible for $\left(n T_{1}\right)^{2}$ and $\left(T_{2}-a_{6} n^{2}\right)^{2}$ to be both zero and $n=0$ can be a solution of (11) if $K_{3}=T_{2}$. If the first bifurcation point is $\left(n_{b}^{0}, \eta_{b}^{0}\right)$, then the other bifurcation points $\left(n_{b}, \eta_{b}\right)$ satisfy $n_{b} \eta_{b}=n_{b}^{0} \eta_{b}^{0}+2 v \pi$, $v=1,2, \ldots, \infty$.

One can notice that if $n$ is a solution of (10) (or (11)), then so is $-n$. Hence, in the following we only investigate for positive solutions $n$ of (10), or (11) respectively. By squaring the two equations into system (10) and then adding them, it follows that

$$
\begin{aligned}
n^{6} & +\left(K_{1}^{2}-2 K_{2}-a_{6}^{2}\right) n^{4} \\
& +\left(K_{2}^{2}-2 K_{1} K_{3}-T_{1}^{2}+2 a_{6} T_{2}\right) n^{2}+K_{3}^{2}-T_{2}^{2}=0 .
\end{aligned}
$$

As $\bar{E}$ is locally asymptotically stable at $\eta=0$, it satisfies the Routh-Hurwitz conditions for stability for a cubic polynomial $[19,20]$. Equation (12) is a cubic in $n^{2}$ and the left-hand side is positive for very large values of $n^{2}$ and also at $n=0$. Suppose that conditions of Lemma 1(I) in [19] are satisfied; that is, (12) has at least one positive real simple root. Moreover, to apply the Hopf bifurcation theorem, according to [19], the following theorem in this situation applies.

Theorem 2. Suppose that $n_{b}$ is the least positive simple root of (12). Then, $i n\left(\eta_{b}\right)=i n_{b}$ is a simple root of (4) (at $\zeta=$ $\xi=0)$ and $m(\eta)+i n(\eta)$ is differentiable with respect to $\eta$ in a neighbourhood of $\eta=\eta_{b}$.

To establish an Andronov-Hopf bifurcation at $\eta=\eta_{b}$, we need to show that the following transversality condition $d m /\left.d \eta\right|_{\eta=\eta_{b}} \neq 0$ is satisfied.

Hence, if we denote

$$
H(\chi, \eta)=\chi^{3}+K_{1} \chi^{2}+K_{2} \chi+K_{3}+\ell^{-\chi \eta}\left(a_{6} \chi^{2}+T_{1} \chi+T_{2}\right)
$$


then

$$
\begin{aligned}
\frac{d \chi}{d \eta}= & -\frac{\partial H / \partial \eta}{\partial H / \partial \chi} \\
= & \chi \ell^{-\chi \eta}\left(a_{6} \chi^{2}+T_{1} \chi+T_{2}\right) \\
& \times\left(3 \chi^{2}+2 K_{1} \chi+K_{2}-\eta \ell\left(a_{6} \chi^{2}+T_{1} \chi+T_{2}\right)\right. \\
& \left.+\ell^{-\chi \eta}\left(2 a_{6} \chi+T_{1}\right)\right)^{-1} .
\end{aligned}
$$

Evaluating the real part of this equation at $\eta=\eta_{b}$ and setting $\chi=i n_{b}$ yield

$$
\begin{aligned}
\left.\frac{d m}{d \eta}\right|_{\eta=\eta_{b}}= & \left.\operatorname{Re}\left(\frac{d \chi}{d \eta}\right)\right|_{\eta=\eta_{b}} \\
= & n_{b}^{2}\left[3 n_{b}^{2}+2\left(K_{1}^{2}-2 K_{2}-a_{6}^{2}\right) n_{b}^{2}\right. \\
& \left.+K_{2}^{2}-2 K_{1} K_{3}+2 a_{6} T_{2}-T_{1}^{2}\right] \times\left(L^{2}+I^{2}\right)^{-1},
\end{aligned}
$$

where $L=-3 n_{b}^{2}+K_{2}+\eta_{b}\left(-K_{1} n_{b}^{2}+K_{3}\right)+T_{1} \cos n_{b} \eta_{b}+$ $2 a_{6} n_{b} \sin n_{b} \eta_{b}$ and $I=2 K_{1} n_{b}+\eta_{b}\left(-n_{b}^{3}+K_{2} n_{b}\right)+$ $2 a_{6} n_{b} \cos n_{b} \eta_{b}-T_{1} \sin n_{b} \eta_{b}$.

Let $\theta=\eta_{b}^{2}$, then (12) reduces to

$$
\begin{aligned}
g(\theta)= & \theta^{3}+\left(K_{1}^{2}-2 K_{2}-a_{6}^{2}\right) \theta^{2} \\
& +\left(K_{2}^{2}-2 K_{1} K_{3}+2 a_{6} T_{2}-T_{1}^{2}\right) \theta+K_{3}^{2}-T_{2}^{2} .
\end{aligned}
$$

Then for $g^{\prime}(\theta)$, we have

$$
\begin{aligned}
\left.g^{\prime}(\theta)\right|_{\eta=\eta_{b}}= & \left.\frac{d g}{d \theta}\right|_{\eta=\eta_{b}} \\
= & 3 \theta^{2}+2\left(K_{1}^{2}-2 K_{2}-a_{6}^{2}\right) \theta+K_{2}^{2} \\
& -2 K_{1} K_{3}+2 a T_{2}-T_{1}^{2} .
\end{aligned}
$$

If $\eta_{b}$ is the least positive simple root of (12), then

$$
\left.\frac{d g}{d \eta}\right|_{\theta=n_{b}^{2}}>0
$$

Hence,

$$
\left.\frac{d m}{d \eta}\right|_{\eta=\eta_{b}}=\left.\operatorname{Re}\left(\frac{d \chi}{d \eta}\right)\right|_{\eta=\eta_{b}}=\frac{n_{b}^{2} g^{\prime}\left(n_{b}^{2}\right)}{L^{2}+I^{2}}>0 .
$$

According to the Hopf bifurcation theorem [21], we define the following theorem.

Theorem 3. If $n_{b}$ is the least positive root of (12), then an Andronov-Hopf bifurcation occurs as $\eta$ passes through $\eta_{b}$.

Corollary 4. When $\eta<\eta_{b}$, then the steady state $\bar{E}$ of system (2) is locally asymptotically stable.

\section{The Case $\zeta=0 ; \xi, \eta>0$}

We return to the study of (4) which with $\xi, \eta>0$ has the form

$$
\begin{aligned}
\chi^{3} & +K_{41} \chi^{2}+K_{5} \chi+K_{6}+\ell^{-\chi \tau_{1}}\left(a_{5} \chi^{2}+T_{3} \chi+T_{4}\right) \\
& +\ell^{-\chi \tau_{2}}\left(a_{6} \chi^{2}+T_{5} \chi+T_{6}\right)+\ell^{-\chi \tau_{3}}\left(a_{12} \chi+T_{7}\right)=0,
\end{aligned}
$$

where $K_{41}=a_{1}+a_{4}, K_{5}=a_{2}+a_{7}, K_{6}=a_{3}+a_{13}, T_{3}=a_{8}+a_{10}$, $T_{4}=a_{14}+a_{16}, T_{5}=a_{9}+a_{11}, T_{6}=a_{15}+a_{17}, T_{7}=a_{18}+a_{19}$, and $\tau=\left[\tau_{1}=\xi, \tau_{2}=\eta, \tau_{3}=\xi+\eta\right]^{T}$ denotes a point in the time delay space; that is, $\tau \in \Omega \subset R_{+}^{3}$. $\Omega$ is the time delay space and $R_{+}^{3}$ denotes the set of nonnegative real numbers. In order to assess the stability of (2) with respect to any delay $\tau$, one should know where all $\chi$ roots of (20) lie on the complex plane. Equation (20) has infinitely many roots on the complex plane due to the transcendental term $\ell^{-\chi \tau}$. This makes the analytical stability assessment intractable.

In Section 2, we obtain that in the absence of delays, $\bar{E}$ is locally asymptotically stable if the conditions (7) are valid. By Remark 1, this implies that $\chi=0$ is not root of (20). Further, we introduce the following simple result (which was proved by [18]) using Rouche's theorem.

Lemma 5. Consider the exponential polynomial $P\left(\chi, \ell^{-\chi \tau_{1}}\right.$, $\left.\ldots, \ell^{-\chi \tau_{m}}\right)=\chi^{n}+p_{1}^{(0)} \chi^{n-1}+\cdots+p_{n-1}^{(0)} \chi+p_{n}^{(0)}+\left[p_{1}^{(1)} \chi^{n-1}+\cdots+\right.$ $\left.p_{n-1}^{(1)} \chi+p_{n}^{(1)}\right] \ell^{-\chi \tau_{1}}+\cdots+\left[p_{1}^{(m)} \chi^{n-1}+\cdots+p_{n-1}^{(m)} \chi+p_{n}^{(m)}\right] \ell^{-\chi \tau_{m}}$, where $\tau_{i} \geq 0(i=1,2, \ldots, m)$ and $p_{j}^{(i)}(i=0,1, \ldots, m ; j=$ $1,2, \ldots, n)$ are constants. As $\left(\tau_{1}, \tau_{2}, \ldots, \tau_{m}\right)$ vary, the sum of the order of the zeros of $P\left(\chi, \ell^{-\chi \tau_{1}}, \ldots, \ell^{-\chi \tau_{m}}\right)$ on the open right half plane can change only if a zero appears there or crosses the imaginary axis.

Obviously, in $(n>0)$ is a root of (20) if and only if $n$ satisfies

$$
\begin{aligned}
-n^{3} i & -K_{41} n^{2}+K_{5} n i+K_{6} \\
& +\left(\cos n \tau_{1}-i \sin n \tau_{1}\right)\left(-a_{5} n^{2}+T_{3} n i+T_{4}\right) \\
& +\left(\cos n \tau_{2}-i \sin n \tau_{2}\right)\left(-a_{6} n^{2}+T_{5} n i+T_{6}\right) \\
& +\left(\cos n \tau_{3}-i \sin n \tau_{3}\right)\left(a_{12} n i+T_{7}\right)=0 .
\end{aligned}
$$

Separating the real and imaginary parts into (21), we obtain

$$
\begin{aligned}
-K_{41} n^{2} & +K_{6}+a_{12} n \sin n \tau_{3}+T_{7} \cos n \tau_{3} \\
= & \left(a_{5} n^{2}-T_{4}\right) \cos n \tau_{1}-T_{3} n \sin n \tau_{1} \\
& +\left(a_{6} n^{2}-T_{6}\right) \cos n \tau_{2}-T_{5} n \sin n \tau_{2}, \\
-n^{3}+ & K_{5} n+a_{12} n \cos n \tau_{3}-T_{7} \sin n \tau_{3} \\
= & \left(T_{4}-a_{5} n^{2}\right) \sin n \tau_{1}-T_{3} n \cos n \tau_{1} \\
& +\left(T_{6}-a_{6} n^{2}\right) \sin n \tau_{2}-T_{5} n \cos n \tau_{2} .
\end{aligned}
$$


We square and add (22), and after simplifying, we get that $\tau$ and $n$ must be among the real solutions of

$$
\begin{aligned}
n^{6}+ & \left(K_{41}^{2}-a_{5}^{2}-a_{6}^{2}-2 K_{5}\right) n^{4} \\
+ & {\left[K_{5}^{2}+a_{12}^{2}-T_{3}^{2}-T_{5}^{2}+2\left(a_{5} T_{4}+a_{6} T_{6}-K_{4} K_{6}\right)\right] n^{2} } \\
+ & K_{6}^{2}+T_{7}^{2} \\
=2[ & \left(K_{41} n^{2}-K_{6}\right)\left(T_{7} \cos n \tau_{3}+a_{12} n \sin n \tau_{3}\right) \\
& +n\left(n^{2}-K_{5}\right)\left(a_{12} n \cos n \tau_{3}-T_{7} \sin n \tau_{3}\right) \\
& +\left(a_{5} n^{2}-T_{4}\right)\left(a_{6} n^{2}-T_{6}\right) \cos n\left(\tau_{1}-\tau_{2}\right) \\
& +T_{5} n\left(a_{5} n^{2}-T_{4}\right) \sin n\left(\tau_{1}-\tau_{2}\right) \\
& +T_{3} n\left(T_{6}-a_{6} n^{2}\right) \sin n\left(\tau_{1}-\tau_{2}\right) \\
& \left.+T_{3} T_{5} n^{2} \cos n\left(\tau_{1}-\tau_{2}\right)\right] .
\end{aligned}
$$

We note that the right-hand side of (23) is always less than $2\left[\left|K_{41} n^{2}-K_{6}\right|\left|T_{7}+a_{12} n\right|+\left|n^{2}-K_{5}\right|\left|a_{12} n-T_{7}\right| n+\mid a_{5} n^{2}-\right.$ $\left.T_{4}|| a_{6} n^{2}-T_{6}|+| T_{5}|| a_{5} n^{2}-T_{4}|n+| T_{3}|| T_{6}-a_{6} n^{2}|n+| T_{3} T_{5} \mid n^{2}\right]$. Hence if the inequality

$$
\begin{aligned}
\omega^{6}+ & \left(K_{41}^{2}-a_{5}^{2}-a_{6}^{2}-2 K_{5}\right) \omega^{4} \\
+ & {\left[K_{5}^{2}+a_{12}^{2}-T_{3}^{2}-T_{5}^{2}+2\left(a_{5} T_{4}+a_{6} T_{6}-K_{41} K_{6}\right)\right] \omega^{2} } \\
+ & K_{6}^{2}+T_{7}^{2} \\
>2 & {\left[\left|T_{3} T_{5}\right| \omega^{2}+\left|K_{41} \omega^{2}-K_{6}\right|\left|T_{7}+a_{12} \omega\right|\right.} \\
& +\left|\omega^{2}-K_{5}\right|\left|a_{12} \omega-T_{7}\right| \omega+\left|a_{5} \omega^{2}-T_{4}\right|\left|a_{6} \omega^{2}-T_{6}\right| \\
& \left.+\left|T_{5}\right|\left|a_{5} \omega^{2}-T_{4}\right| \omega+\left|T_{3}\right|\left|T_{6}-a_{6} \omega^{2}\right| \omega\right]
\end{aligned}
$$

has no real solution on $0<\omega<n_{+}$, then (23) cannot be satisfied. Note that $n_{+}$is the positive solution of first equation in (6) (when $m=0$ and $\zeta=0$ ), which we write as

$$
\begin{aligned}
&\left(a_{1}+a_{4}\right) n^{2} \\
&=\psi(n) \\
&=\left[a_{3}+a_{13}-\left(a_{5} \cos n \xi+a_{6} \cos n \eta\right) n^{2}\right. \\
& \quad+\left(a_{8} \sin n \xi+a_{9} \sin n \eta+a_{10} \sin n \xi\right. \\
&\left.\quad+a_{11} \sin n \eta+a_{12} \sin n(\xi+\eta)\right) n \\
&+a_{14} \cos n \xi+a_{15} \cos n \eta+a_{16} \cos n \xi \\
&+\left.+a_{17} \cos n \eta+a_{18} \cos n(\xi+\eta)+a_{19} \cos n(\xi+\eta)\right]
\end{aligned}
$$

$$
\begin{aligned}
& \leq a_{3}+a_{13}+\left|a_{14}\right|+\left|a_{15}\right|+\left|a_{16}\right|+\left|a_{17}\right| \\
&+\left|a_{18}\right|+\left|a_{19}\right|+\left(\left|a_{8}\right|+\left|a_{9}\right|+\left|a_{10}\right|+\left|a_{11}\right|+\left|a_{12}\right|\right) n \\
&-\left(\left|a_{5}\right|+\left|a_{6}\right|\right) n^{2}, \text { i.e., } \\
&\left(a_{1}+a_{4}+\left|a_{5}\right|+\left|a_{6}\right|\right) n^{2}-\left(\left|a_{8}\right|+\left|a_{9}\right|+\left|a_{10}\right|+\left|a_{11}\right|+\left|a_{12}\right|\right) n \\
& \quad-\left(a_{3}+a_{13}+\cdots+\left|a_{19}\right|\right)=0 .
\end{aligned}
$$

Thus, for $n_{+}$, we have

$$
n_{+}=\frac{1}{2 a}\left(b+\sqrt{b^{2}+4 a c}\right),
$$

where $a=a_{1}+a_{4}+\left|a_{5}\right|+\left|a_{6}\right| \neq 0, b=\left|a_{8}\right|+\cdots+\left|a_{12}\right|$ and $c=a_{3}+a_{13}+\left|a_{14}\right|+\cdots+\left|a_{19}\right|$. It is clear that $n \leq n_{+}$.

Rearranging terms, we write (24) as

$$
\begin{aligned}
\left(\left|K_{41} \omega^{2}-K_{6}\right|-\left|T_{7}+a_{12} \omega\right|\right)^{2} \\
+\left(\omega\left|\omega^{2}-K_{5}\right|-\left|a_{12} \omega-T_{7}\right|\right)^{2} \\
\quad+\left(\left|a_{5}\right| \omega^{2}-\left|T_{4}\right|\right)^{2}+\left(\left|a_{6}\right| \omega^{2}-\left|T_{6}\right|\right)^{2} \\
\quad+\left(T_{3}^{2}+T_{5}^{2}\right) \omega^{2}>\left(\left|a_{5} \omega^{2}-T_{4}\right|+\left|a_{6} \omega^{2}-T_{6}\right|\right)^{2} \\
+\left(\left|a_{5} \omega^{2}-T_{4}\right|+\left|T_{5}\right| \omega\right)^{2}+\left(\left|T_{3}\right| \omega+\left|T_{6}-a_{6} \omega^{2}\right|\right)^{2} \\
\quad+\left(\left|T_{3}\right|+\left|T_{5}\right|\right)^{2} \omega^{2}+a_{12}^{2} \omega^{2}+T_{4}^{2}+T_{6}^{2}+T_{7}^{2} .
\end{aligned}
$$

Hence, the following theorem can be formulated.

Theorem 6. Let $a_{3}+a_{13}+\left|a_{14}\right|+\cdots+\left|a_{19}\right| \neq 0$ and (27) hold. Then there is no change in stability of $\bar{E}$.

Remark 7. In the special case that $\xi=\eta$, the characteristic equation (20) becomes

$$
\begin{aligned}
\chi^{3}+ & K_{41} \chi^{2}+K_{5} \chi+K_{6}+\ell^{-\chi \tau_{1}}\left(T_{56} \chi^{2}+T_{35} \chi+T_{46}\right) \\
& +\ell^{-2 \chi \tau_{1}}\left(a_{12} \chi+T_{7}\right)=0
\end{aligned}
$$

where $T_{56}=a_{5}+a_{6}, T_{35}=T_{3}+T_{5}$ and $T_{46}=T_{4}+T_{6}$. Therefore, this case is a private one of Theorem 6 .

Corollary 8. If conditions of Theorem 6 are not valid and $\tau_{2}^{b i f}$ is defined as in Theorem 3, then according to Lemma 5 for any $\tau_{2} \in\left[0, \tau_{b}\right)$, there exists a $\tau_{3}^{\text {bif }}\left(\tau_{2}\right)>0\left(\tau_{1}^{b i f}\left(\tau_{2}\right)>0\right.$ resp. $)$ such that the steady state $\bar{E}$ of system (2) is unstable when $\tau_{3} \in$ $\left[0, \tau_{3}^{b i f}\left(\tau_{2}\right)\right)\left(\tau_{1} \in\left[0, \tau_{1}^{b i f}\left(\tau_{2}\right)\right)\right.$ resp. $)$, and an Andronov-Hopf bifurcation takes place. 


\section{The General Case $\zeta, \xi, \eta>0$}

Similar to Section 4 , we set that $\chi=i n(n>0)$ is a root of $(4)$ if and only if $n$ satisfies

$$
\begin{aligned}
-n^{3} i & -a_{1} n^{2}+a_{2} n i+a_{3} \\
& +\left(\cos n \tau_{1}-i \sin n \tau_{1}\right)\left(-a_{4} n^{2}+a_{7} n i+a_{13}\right) \\
& +\left(\cos n \tau_{2}-i \sin n \tau_{2}\right)\left(-a_{5} n^{2}+a_{8} n i+a_{14}\right) \\
& +\left(\cos n \tau_{3}-i \sin n \tau_{3}\right)\left(-a_{6} n^{2}+a_{9} n i+a_{15}\right) \\
& +\left(\cos n \tau_{4}-i \sin n \tau_{4}\right)\left(a_{6} n i+a_{16}\right) \\
& +\left(\cos n \tau_{5}-i \sin n \tau_{5}\right)\left(a_{11} n i+a_{17}\right) \\
& +\left(\cos n \tau_{6}-i \sin n \tau_{6}\right)\left(a_{12} n i+a_{18}\right) \\
& +a_{19}\left(\cos n \tau_{7}-i \sin n \tau_{7}\right)=0
\end{aligned}
$$

where $\tau=\left[\tau_{1}=\zeta, \tau_{2}=\xi, \tau_{3}=\eta, \tau_{4}=\zeta+\xi, \tau_{5}=\zeta+\eta\right.$, $\left.\tau_{6}=\xi+\eta, \tau_{7}=\zeta+\xi+\eta\right]^{T}$ denotes a point in the time delay space; that is, $\tau \in \Omega \subset R_{+}^{7}$. $\Omega$ is the time delay space and $R_{+}^{7}$ denotes the set of nonnegative real numbers.

Separating the real and imaginary parts into (29), we have

$$
\begin{gathered}
-a_{1} n^{2}+a_{3}-A_{1}-B_{1}-C_{1}=-D_{1}-E_{1}-F_{1}-a_{19} \cos n \tau_{7}, \\
-n^{3}+a_{2} n+A_{2}+B_{2}+C_{2}=D_{2}+E_{2}+F_{2}+a_{19} \sin n \tau_{7},
\end{gathered}
$$

where

$$
\begin{aligned}
& A_{1}=\left(a_{4} n^{2}-a_{13}\right) \cos n \tau_{1}-a_{7} n \sin n \tau_{1}, \\
& B_{1}=\left(a_{5} n^{2}-a_{14}\right) \cos n \tau_{2}-a_{8} n \sin n \tau_{2}, \\
& C_{1}=\left(a_{6} n^{2}-a_{15}\right) \cos n \tau_{3}-a_{9} n \sin n \tau_{3}, \\
& D_{1}=a_{10} n \sin n \tau_{4}+a_{16} \cos n \tau_{4}, \\
& E_{1}=a_{11} n \sin n \tau_{5}+a_{17} \cos n \tau_{5}, \\
& F_{1}=a_{12} n \sin n \tau_{6}+a_{18} \cos n \tau_{6}, \\
& A_{2}=\left(a_{4} n^{2}-a_{13}\right) \sin n \tau_{1}+a_{7} n \cos n \tau_{1}, \\
& B_{2}=\left(a_{5} n^{2}-a_{14}\right) \sin n \tau_{2}+a_{8} n \cos n \tau_{2}, \\
& C_{2}=\left(a_{6} n^{2}-a_{15}\right) \sin n \tau_{3}+a_{9} n \cos n \tau_{3}, \\
& D_{2}=a_{10} n \cos n \tau_{4}-a_{16} \sin n \tau_{4}, \\
& E_{2}=a_{11} n \cos n \tau_{5}-a_{17} \sin n \tau_{5}, \\
& F_{2}=a_{12} n \cos n \tau_{6}-a_{18} \sin n \tau_{6} .
\end{aligned}
$$

Adding up the squares of both equations into (30), we have

$$
\begin{aligned}
n^{6}+ & S_{1} n^{4}+S_{2} n^{2}+S_{3} \\
=2 & \left\{\left(a_{4} n^{2}-a_{13}\right)\right. \\
& \times\left[\left(a_{3}-a_{1} n^{2}\right) \cos n \tau_{1}-\left(-n^{3}+a_{2} n\right) \sin n \tau_{1}\right]
\end{aligned}
$$

$$
\begin{aligned}
& +a_{7} n\left[\left(a_{3}-a_{1} n^{2}\right) \sin n \tau_{1}+\left(-n^{3}+a_{2} n\right) \cos n \tau_{1}\right] \\
& +a_{19}\left(a_{12} n \sin n \tau_{1}+a_{18} \cos n \tau_{1}\right) \\
& +\left(a_{5} n^{2}-a_{14}\right) \\
& \times\left[\left(a_{3}-a_{1} n^{2}\right) \cos n \tau_{2}-\left(-n^{3}+a_{2} n\right) \sin n \tau_{2}\right] \\
& +a_{19}\left(a_{11} n \sin n \tau_{2}+a_{17} \cos n \tau_{2}\right) \\
& +a_{8} n\left[\left(a_{3}-a_{1} n^{2}\right) \sin n \tau_{2}+\left(-n^{3}+a_{2} n\right) \cos n \tau_{2}\right] \\
& +a_{19}\left(a_{10} n \sin n \tau_{3}+a_{16} \cos n \tau_{3}\right) \\
& +\left(a_{6} n^{2}-a_{15}\right) \\
& \times\left[\left(a_{3}-a_{1} n^{2}\right) \cos n \tau_{3}-\left(-n^{3}+a_{2} n\right) \sin n \tau_{3}\right] \\
& +a_{9} n\left[\left(a_{3}-a_{1} n^{2}\right) \sin n \tau_{3}+\left(-n^{3}+a_{2} n\right) \cos n \tau_{3}\right] \\
& +\left(a_{5} n^{2}-a_{14}\right)\left[a_{7} n \sin n\left(\tau_{1}-\tau_{2}\right)\right. \\
& \left.-\left(a_{4} n^{2}-a_{13}\right) \cos n\left(\tau_{1}-\tau_{2}\right)\right] \\
& +a_{17}\left[a_{18} \cos n\left(\tau_{1}-\tau_{2}\right)-a_{12} n \sin n\left(\tau_{1}-\tau_{2}\right)\right] \\
& +a_{11} n\left[a_{12} n \cos n\left(\tau_{1}-\tau_{2}\right)+a_{18} \sin n\left(\tau_{1}-\tau_{2}\right)\right] \\
& +\left(a_{6} n^{2}-a_{15}\right)\left[a_{7} n \sin n\left(\tau_{1}-\tau_{3}\right)\right. \\
& \left.-\left(a_{4} n^{2}-a_{13}\right) \cos n\left(\tau_{1}-\tau_{3}\right)\right] \\
& +a_{10} n\left[a_{12} n \cos n\left(\tau_{1}-\tau_{3}\right)+a_{18} \sin n\left(\tau_{1}-\tau_{3}\right)\right] \\
& +a_{16}\left[a_{18} \cos n\left(\tau_{1}-\tau_{3}\right)-a_{12} n \sin n\left(\tau_{1}-\tau_{3}\right)\right] \\
& -a_{9} n\left[a_{7} n \cos n\left(\tau_{1}-\tau_{3}\right)\right. \\
& \left.+\left(a_{4} n^{2}-a_{13}\right) \sin n\left(\tau_{1}-\tau_{3}\right)\right] \\
& +\left(a_{6} n^{2}-a_{15}\right)\left[a_{8} n \sin n\left(\tau_{2}-\tau_{3}\right)\right. \\
& \left.-\left(a_{5} n^{2}-a_{14}\right) \cos n\left(\tau_{2}-\tau_{3}\right)\right] \\
& +a_{10} n\left[a_{11} n \cos n\left(\tau_{2}-\tau_{3}\right)+a_{17} \sin n\left(\tau_{2}-\tau_{3}\right)\right] \\
& -a_{16}\left[a_{11} n \sin n\left(\tau_{2}-\tau_{3}\right)-a_{17} \cos n\left(\tau_{2}-\tau_{3}\right)\right] \\
& -a_{9} n\left[a_{8} n \cos n\left(\tau_{2}-\tau_{3}\right)\right. \\
& \left.\left.+\left(a_{5} n^{2}-a_{14}\right) \sin n\left(\tau_{2}-\tau_{3}\right)\right]\right\} \\
& +4 a_{19}\left\{\operatorname { s i n } n ( \tau _ { 1 } + \tau _ { 2 } ) \left[a_{10} n \cos n\left(\tau_{1}+\tau_{2}+\tau_{3}\right)\right.\right. \\
& \left.-a_{16} \sin n\left(\tau_{1}+\tau_{2}+\tau_{3}\right)\right] \\
& +\sin n\left(\tau_{1}+\tau_{3}\right)\left[a_{11} n \cos n\left(\tau_{1}+\tau_{2}+\tau_{3}\right)\right. \\
& \left.-a_{17} \sin n\left(\tau_{1}+\tau_{2}+\tau_{3}\right)\right] \\
& +\sin n\left(\tau_{2}+\tau_{3}\right)\left[a_{12} n \cos n\left(\tau_{1}+\tau_{2}+\tau_{3}\right)\right. \\
& \left.\left.-a_{18} \sin n\left(\tau_{1}+\tau_{2}+\tau_{3}\right)\right]\right\},
\end{aligned}
$$


where

$$
\begin{gathered}
\tau_{4}+\tau_{7}=2\left(\tau_{1}+\tau_{2}\right)+\tau_{3}, \\
\tau_{5}+\tau_{6}=\tau_{1}+\tau_{2}+2 \tau_{3}, \\
\tau_{5}+\tau_{7}=\tau_{2}+2\left(\tau_{1}+\tau_{3}\right), \\
\tau_{6}+\tau_{7}=\tau_{1}+2\left(\tau_{2}+\tau_{3}\right), \\
\tau_{4}-\tau_{5}=\tau_{2}-\tau_{3}, \quad \tau_{4}-\tau_{6}=\tau_{1}-\tau_{3}, \\
\tau_{5}-\tau_{6}=\tau_{1}-\tau_{2}, \\
S_{1}=a_{1}^{2}+a_{4}^{2}+a_{5}^{2}+a_{6}^{2}-2 a_{2}, \\
S_{2}=a_{2}^{2}+a_{7}^{2}+a_{8}^{2}+a_{9}^{2}-a_{10}^{2}-a_{11}^{2}-a_{12}^{2}, \\
S_{3}=a_{3}^{2}+a_{13}^{2}+a_{14}^{2}+a_{15}^{2}-a_{16}^{2}-a_{17}^{2}-a_{18}^{2}-a_{19}^{2} .
\end{gathered}
$$

Clearly, the right-hand side of (32) is always less than

$$
\begin{aligned}
2\left\{\left|n^{3}-a_{1} n^{2}-a_{2} n+a_{3}\right|\right. & \\
\quad & {\left[\left|a_{4} n^{2}-a_{13}\right|+\left|a_{5} n^{2}-a_{14}\right|+\left|a_{6} n^{2}-a_{15}\right|\right] } \\
& +a_{19}\left[\left|a_{12} n+a_{18}\right|+\left|a_{11} n+a_{17}\right|+\left|a_{10} n+a_{16}\right|\right] \\
& +n\left|-n^{3}-a_{1} n^{2}+a_{2} n+a_{3}\right|\left(a_{7}+a_{8}+a_{9}\right) \\
& +n\left(a_{10}+a_{11}\right)\left|a_{12} n+a_{18}\right|-\left(a_{16}+a_{17}\right)\left|a_{12} n-a_{18}\right| \\
& +\left|-a_{4} n^{2}+a_{7} n+a_{13}\right|\left[\left|a_{5} n^{2}-a_{14}\right|+\left|a_{6} n^{2}-a_{15}\right|\right] \\
& -n\left(a_{8}+a_{9}\right)\left|a_{4} n^{2}+a_{7} n-a_{13}\right|-a_{9} n\left|a_{5} n^{2}+a_{8} n-a_{14}\right| \\
& +\left|a_{6} n^{2}-a_{15}\right|\left|-a_{5} n^{2}+a_{8} n+a_{14}\right| \\
& \left.+a_{10} n\left|a_{11} n+a_{17}\right|-a_{16}\left|a_{11} n-a_{17}\right|\right\} \\
+ & 4 a_{19}\left[\left|a_{10} n-a_{16}\right|+\left|a_{11} n-a_{17}\right|+\left|a_{12} n-a_{18}\right|\right] .
\end{aligned}
$$

Hence, if the inequality

$$
\begin{aligned}
\lambda^{6}+S_{1} & \lambda^{4}+S_{2} \lambda^{2}+S_{3} \\
>2\{ & \left\{\left|\lambda^{3}-a_{1} \lambda^{2}-a_{2} \lambda+a_{3}\right|\right. \\
& \times\left[\left|a_{4} \lambda^{2}-a_{13}\right|+\left|a_{5} \lambda^{2}-a_{14}\right|+\left|a_{6} \lambda^{2}-a_{15}\right|\right] \\
& +a_{19}\left[\left|a_{12} \lambda+a_{18}\right|+\left|a_{11} \lambda+a_{17}\right|+\left|a_{10} \lambda+a_{16}\right|\right] \\
& +\left(a_{7}+a_{8}+a_{9}\right)\left|-\lambda^{3}-a_{1} \lambda^{2}+a_{2} \lambda+a_{3}\right| \lambda \\
& +\left(a_{10}+a_{11}\right)\left|a_{12} \lambda+a_{18}\right| \lambda-\left(a_{16}+a_{17}\right)\left|a_{12} \lambda-a_{18}\right| \\
& +\left|-a_{4} \lambda^{2}+a_{7} \lambda+a_{13}\right|\left[\left|a_{5} \lambda^{2}-a_{14}\right|+\left|a_{6} \lambda^{2}-a_{15}\right|\right]
\end{aligned}
$$

$$
\begin{aligned}
& -\left(a_{8}+a_{9}\right)\left|a_{4} \lambda^{2}+a_{7} \lambda-a_{13}\right| \lambda \\
& -a_{9} \lambda\left|a_{5} \lambda^{2}+a_{8} \lambda-a_{14}\right| \\
& +\left|a_{6} \lambda^{2}-a_{15}\right|\left|-a_{5} \lambda^{2}+a_{8} \lambda+a_{14}\right| \\
& \left.+a_{10} \lambda\left|a_{11} \lambda+a_{17}\right|-a_{16}\left|a_{11} \lambda-a_{17}\right|\right\} \\
+ & 4 a_{19}\left[\left|a_{10} \lambda-a_{16}\right|+\left|a_{11} \lambda-a_{17}\right|+\left|a_{12} \lambda-a_{18}\right|\right]
\end{aligned}
$$

has no real solution on $0<\lambda<n_{+}$, then (32) cannot be satisfied. Similar to Section 4 , we note that $n_{+}$is the positive solution of first equation in (6) (when $m=0$ ), which is written as

$$
\begin{aligned}
a_{1} n^{2}= & \Psi(n) \\
= & {\left[a_{3}-\left(a_{4} \cos n \zeta+a_{5} \cos n \xi+a_{6} \cos n \eta\right) n^{2}\right.} \\
& +\left(a_{7} \sin n \zeta+a_{8} \sin n \xi+a_{9} \sin n \eta+a_{10} \sin n(\zeta+\xi)\right. \\
& \left.+a_{11} \sin n(\zeta+\eta)+a_{12} \sin n(\xi+\eta)\right) n+a_{13} \cos n \zeta \\
& +a_{14} \cos n \xi+a_{15} \cos n \eta+a_{16} \cos n(\zeta+\xi) \\
& +a_{17} \cos n(\zeta+\eta)+a_{18} \cos n(\xi+\eta) \\
& \left.+a_{19} \cos n(\zeta+\xi+\eta)\right] \\
\leq & a_{3}+\left|a_{13}\right|+\left|a_{14}\right|+\left|a_{15}\right|+\left|a_{16}\right|+\left|a_{17}\right|+\left|a_{18}\right| \\
& +\left|a_{19}\right|+\left(\left|a_{7}\right|+\left|a_{8}\right|+\left|a_{9}\right|+\left|a_{10}\right|+\left|a_{11}\right|+\left|a_{12}\right|\right) n \\
& -\left(\left|a_{4}\right|+\left|a_{5}\right|+\left|a_{6}\right|\right) n^{2}, \text { i.e. }, \\
\left(a_{1}\right. & \left.+\left|a_{4}\right|+\left|a_{5}\right|+\left|a_{6}\right|\right) n^{2} \\
& -\left(\left|a_{7}\right|+\left|a_{8}\right|+\left|a_{9}\right|+\left|a_{10}\right|+\left|a_{11}\right|+\left|a_{12}\right|\right) n \\
& -\left(a_{3}+\left|a_{13}\right|+\cdots+\left|a_{19}\right|\right)=0 .
\end{aligned}
$$

Thus, for $n_{+}$, we have

$$
n_{+}=\frac{1}{2 A}\left(B+\sqrt{B^{2}+4 A C}\right),
$$

where $A=a_{1}+\left|a_{4}\right|+\left|a_{5}\right|+\left|a_{6}\right| \neq 0, B=\left|a_{7}\right|+\cdots+\left|a_{12}\right|$ and $C=a_{3}+\left|a_{13}\right|+\cdots+\left|a_{19}\right|$. It is clear that $n \leq n_{+}$.

Rearranging terms, we write (35) as

$$
\begin{aligned}
\left(\mid \lambda^{3}-\right. & \left.a_{1} \lambda^{2}-a_{2} \lambda+a_{3}|-| a_{4} \lambda^{2}-a_{13} \mid\right)^{2} \\
& +\left(\left|\lambda^{3}-a_{1} \lambda^{2}-a_{2} \lambda+a_{3}\right|-\left|a_{5} \lambda^{2}-a_{14}\right|\right)^{2} \\
& +\left(a_{11} \lambda-\left|a_{12} \lambda+a_{18}\right|\right)^{2} \\
& +\left(\left|\lambda^{3}-a_{1} \lambda^{2}-a_{2} \lambda+a_{3}\right|-\left|a_{6} \lambda^{2}-a_{15}\right|\right)^{2} \\
& +\left(\left|-\lambda^{3}-a_{1} \lambda^{2}+a_{2} \lambda+a_{3}\right|-\lambda\left|a_{7}+a_{8}+a_{9}\right|\right)^{2} \\
& +\left(a_{17}+\left|a_{12} \lambda-a_{18}\right|\right)^{2}
\end{aligned}
$$




$$
\begin{aligned}
& +\left(\left|a_{6} \lambda^{2}-a_{15}\right|-\left|-a_{5} \lambda^{2}+a_{8} \lambda+a_{14}\right|\right)^{2} \\
& +\left(a_{9} \lambda+\left|a_{5} \lambda^{2}+a_{8} \lambda-a_{14}\right|\right)^{2} \\
& +\lambda\left(1+\left|a_{1} \lambda^{4}+a_{2} a_{3}\right|\right)^{2}+\left(a_{16}-\left|a_{12} \lambda-a_{18}\right|\right)^{2} \\
& +\left(\left|-a_{4} \lambda^{2}+a_{7} \lambda+a_{13}\right|-\left|a_{5} \lambda^{2}-a_{14}\right|\right)^{2} \\
& +\left(\left|-a_{4} \lambda^{2}+a_{7} \lambda+a_{13}\right|-\left|a_{6} \lambda^{2}-a_{15}\right|\right)^{2} \\
& +\left(a_{10} \lambda-\left|a_{12} \lambda+a_{18}\right|\right)^{2} \\
& +\left(\left|a_{4} \lambda^{2}+a_{7} \lambda-a_{13}\right|+\lambda\left|a_{8}+a_{9}\right|\right)^{2} \\
& +\lambda\left(\left|a_{3}+a_{1} a_{2}\right|-\lambda^{2}\right)^{2} \\
& +\lambda\left(\left|a_{1} a_{3}+a_{4} a_{13}\right|+\lambda\right)^{2}+\left(a_{10} \lambda-\left|a_{11} \lambda+a_{17}\right|\right)^{2} \\
& +\left(a_{16}+\left|a_{11} \lambda-a_{17}\right|\right)^{2}+\left(2 a_{19}-\left|a_{10} \lambda-a_{16}\right|\right)^{2} \\
& +\left(2 a_{19}-\left|a_{11} \lambda-a_{17}\right|\right)^{2}+\left(a_{5}+a_{14} \lambda^{2}\right)^{2} \\
& +\left(a_{6}+a_{15} \lambda^{2}\right)^{2}+\left(a_{8}-a_{9} \lambda^{2}\right)^{2} \\
& +a_{7}^{2} \lambda^{2}>2\left(\lambda^{3}-a_{1} \lambda^{2}-a_{2} \lambda+a_{3}\right)^{2} \\
& +\left(a_{5} \lambda^{2}-a_{14}\right)^{2}+2\left(a_{6} \lambda^{2}-a_{15}\right)^{2} \\
& +\left(-\lambda^{3}-a_{1} \lambda^{2}+a_{2} \lambda+a_{3}\right)^{2} \\
& +\lambda\left(a_{7}+a_{8}+a_{9}\right)^{2}+\left(-a_{5} \lambda^{2}+a_{8} \lambda+a_{14}\right)^{2} \\
& +\left(a_{5} \lambda^{2}+a_{8} \lambda-a_{14}\right)^{2} \\
& +\lambda\left[\left(a_{1} \lambda^{4}+a_{2} a_{3}\right)^{2}+\left(a_{1} a_{2}+a_{3}\right)^{2}\right. \\
& \left.+\lambda^{4}+\left(a_{1} a_{3}+a_{4} a_{13}\right)^{2}+\lambda^{2}\right] \\
& +2\left(-a_{4} \lambda^{2}+a_{7} \lambda+a_{13}\right)^{2}+\left(a_{4} \lambda^{2}+a_{7} \lambda-a_{13}\right)^{2} \\
& +2\left(a_{10} \lambda-a_{16}\right)^{2}+2\left(a_{11} \lambda-a_{17}\right)^{2} \\
& +\left(a_{19}+\left|a_{12} \lambda+a_{18}\right|\right)^{2}+\left(a_{19}+\left|a_{11} \lambda+a_{17}\right|\right)^{2} \\
& +\left(a_{19}+\left|a_{10} \lambda+a_{16}\right|\right)^{2}+\left(2 a_{19}+\left|a_{12} \lambda-a_{18}\right|\right)^{2} \\
& +\left(a_{9}^{2}+a_{14}^{2}+a_{15}^{2}\right) \lambda^{4}+\left(a_{9}^{2}+3 a_{12}^{2}\right) \lambda^{2} \\
& +a_{5}^{2}+a_{6}^{2}+a_{8}^{2}+a_{10}^{2}+a_{16}^{2}+2 a_{17}^{2}+3 a_{18}^{2}+2 a_{19}^{2} \text {. }
\end{aligned}
$$

Therefore, the following theorem can be formulated.

Theorem 9. Let $a_{3}+\left|a_{13}\right|+\left|a_{14}\right|+\cdots+\left|a_{19}\right| \neq 0$ and (38) hold. Then there is no change in stability of $\bar{E}$.
Remark 10. In the special case that $\zeta=\xi=\eta$, the characteristic equation (4) becomes

$$
\begin{aligned}
& \chi^{3}+a_{1} \chi^{2}+a_{2} \chi+a_{3} \\
& +\ell^{-\chi \eta}\left[\left(a_{4}+a_{5}+a_{6}\right) \chi^{2}\right. \\
& \left.\quad+\left(a_{7}+a_{8}+a_{9}\right) \chi+a_{13}+a_{14}+a_{15}\right] \\
& \quad+\ell^{-2 \chi \eta}\left[\left(a_{10}+a_{11}+a_{12}\right) \chi+a_{16}+a_{17}+a_{18}\right] \\
& +a_{19} \ell^{-3 \chi \eta}=0 .
\end{aligned}
$$

Hence, this case is a private one of Theorem 9.

Corollary 11. If conditions of Theorem 9 are not valid and $\tau_{3}^{\text {bif }}$ is defined as in Theorem 3, then according to Lemma 5 for any $\tau_{3} \in\left[0, \tau_{b}\right)$, there exists a $\tau_{1}^{\text {bif }}\left(\tau_{3}\right)>0\left(\tau_{2}^{\text {bif }}\left(\tau_{3}\right)>0\right.$; $\tau_{4}^{b i f}\left(\tau_{3}\right)>0 ; \tau_{5}^{b i f}\left(\tau_{3}\right)>0 ; \tau_{6}^{b i f}\left(\tau_{3}\right)>0 ; \tau_{7}^{b i f}\left(\tau_{3}\right)>0$ resp.) such that the steady state $\bar{E}$ of system (2) is unstable when $\tau_{1} \in\left[0, \tau_{1}^{\text {bif }}\left(\tau_{3}\right)\right)\left(\tau_{2} \in\left[0, \tau_{2}^{\text {bif }}\left(\tau_{3}\right)\right) ; \tau_{4} \in\left[0, \tau_{4}^{\text {bif }}\left(\tau_{3}\right)\right)\right.$; $\tau_{5} \in\left[0, \tau_{5}^{b i f}\left(\tau_{3}\right)\right) ; \tau_{6} \in\left[0, \tau_{6}^{b i f}\left(\tau_{3}\right)\right) \tau_{7} \in\left[0, \tau_{7}^{b i f}\left(\tau_{3}\right)\right)$ resp. $)$, and an Andronov-Hopf bifurcation take place.

\section{Application: Numerical Analysis}

In this section, we apply the material and results of the previous sections to a nonlinear mathematical model with three discrete time delays, which investigate the dynamics of the gene expression regulated by the miRNA. For a general discussion of the model, we refer the reader to [22, 23]. Hence, we consider the following system:

$$
\begin{gathered}
\frac{d y_{1}}{d t}=\frac{k_{1}}{k_{2}+k_{3} y_{2}^{n_{1}}\left(t-\tau_{1}\right)}-\gamma_{1} y_{1}-k_{4} y_{1} y_{3}, \\
\frac{d y_{2}}{d t}=k_{5} y_{1}\left(t-\tau_{2}\right)-\gamma_{2} y_{2} \\
\frac{d y_{3}}{d t}=l-k_{6} y_{3}\left(t-\tau_{3}\right)-k_{4} y_{1} y_{3}
\end{gathered}
$$

where the protein, $y_{2}$, controls its own synthesis through the repression of mRNA, $y_{1}, y_{3}$ is the concentration of miRNA, $\tau_{1}$ is the time delay for translation, $\tau_{2}$ is the time delay for transcription, $\tau_{3}$ is the average time delay for degradation of miRNA, $n_{1}$ is often referred as a Hill coefficient or a cooperativity coefficient, $k_{i}(i=1, \ldots, 6)$, and $\gamma_{1}$ and $\gamma_{2}$ are the kinetic rate constants. According to $[2,24]$, we consider one representative value for Hill coefficient, $n_{1}=2$. Model (40) is a modified version of a model considered in [22]; that is, in the present paper our model contains three time delays.

The fixed points of the system, $\bar{E}=\left(\bar{y}_{1}, \bar{y}_{2}, \bar{y}_{3}\right)$, represented by (40) can be analytically estimated and are defined 


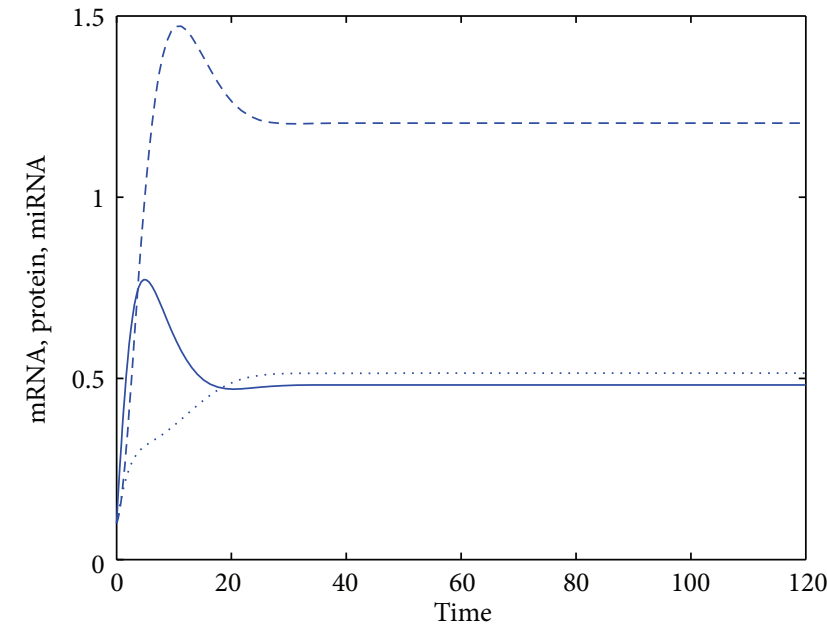

(a)

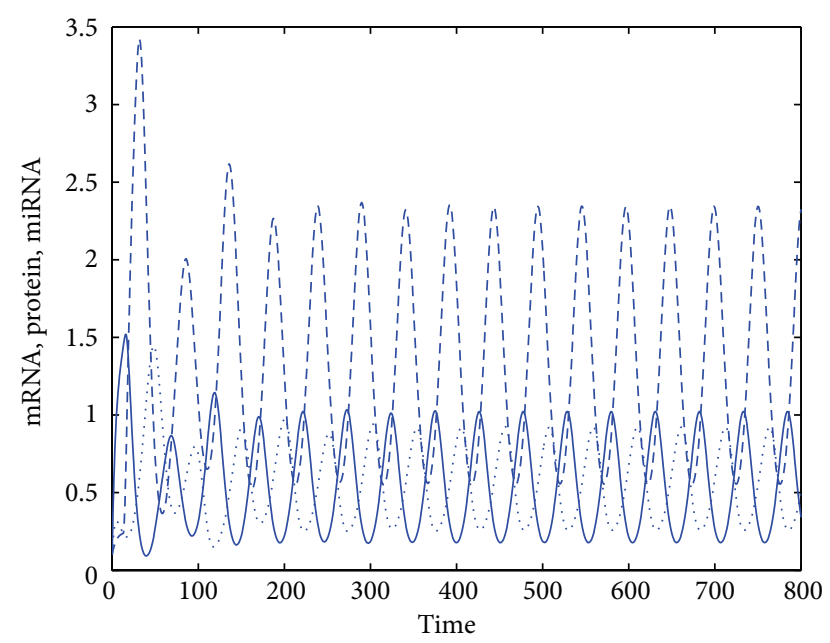

(b)

FIgURE 1: (a) Stable regime of system (40) for $\tau_{1}=\tau_{2}=\tau_{3}=0$ and (b) unstable regime (sustained oscillations) for $\tau_{1}=1, \tau_{2}=13, \tau_{3}=60$.

by the following set of algebraic equations, including the rate constants of the model:

$$
\begin{gathered}
\bar{y}_{2}^{4}+\frac{k_{5}\left(k_{4} l+k_{6} \gamma_{1}\right)}{k_{4} \gamma_{1} \gamma_{2}} \bar{y}_{2}^{3}+\frac{k_{2}}{k_{3}} \bar{y}_{2}^{2} \\
+\frac{k_{5}}{k_{3} k_{4} \gamma_{1} \gamma_{2}}\left[k_{2}\left(k_{4} l+k_{6} \gamma_{1}\right)-k_{1} k_{4}\right] \bar{y}_{2}-\frac{k_{1} k_{5}^{2} k_{6}}{k_{3} k_{4} \gamma_{1} \gamma_{2}^{2}}=0, \\
\bar{y}_{1}=\frac{\gamma_{2}}{k_{5}} \bar{y}_{2}, \quad \bar{y}_{3}=\frac{k_{5} l}{k_{5} k_{6}+\gamma_{2} k_{4} \bar{y}_{2}} .
\end{gathered}
$$

According to Descarte's rule [19, 25], the first equation in (41) has only one real positive root, which ensures that the system has only one physiologically feasible fixed point.

Let us consider a small perturbation around the fixed point $\bar{E}$ of the system (40) defined as

$$
y_{k}=\bar{y}_{k}+x_{k} \quad(k=1,2,3) .
$$

In the case when $n_{1}=2$, the function $k_{1} /\left(k_{2}+k_{3} y_{2}^{2}\left(t-\tau_{1}\right)\right)$ can be written as a MacLaurin series

$$
\begin{aligned}
& \frac{k_{1}}{k_{2}+k_{3} y_{2}^{2}\left(t-\tau_{1}\right)} \\
& =\frac{k_{1}}{\delta+k_{3} \varphi}=\frac{k_{1}}{\delta\left(\left(k_{3} / \delta \varphi\right)+1\right)} \\
& =\frac{k_{1}}{\delta}\left(1-\frac{k_{3}}{\delta} \varphi+\left(\frac{k_{3}}{\delta}\right)^{2} \varphi^{2}-\left(\frac{k_{3}}{\delta}\right)^{3} \varphi^{3}+\cdots\right) \text {, }
\end{aligned}
$$

where $\delta=k_{2}+k_{3} \bar{y}_{2}^{2}$ and $\varphi=2 \bar{y}_{2} x_{2}\left(t-\tau_{1}\right)+x_{2}^{2}\left(t-\tau_{1}\right)$. If we take only linear term from (43) and after substitution of (42) into differential equation (40), we have,

$$
\begin{gathered}
\frac{d x_{1}}{d t}=-c_{1} x_{1}-c_{2} \ell^{-\chi \tau_{1}} x_{2}-c_{3} x_{3}-c_{4} \ell^{-2 \chi \tau_{1}} x_{2}^{2}-k_{4} x_{1} x_{3} \\
\frac{d x_{2}}{d t}=k_{5} \ell^{-\chi \tau_{2}} x_{1}-\gamma_{2} x_{2} \\
\frac{d x_{3}}{d t}=-c_{5} x_{1}-\left(k_{6} \ell^{-\chi \tau_{3}}+c_{3}\right) x_{3}-k_{4} x_{1} x_{3}
\end{gathered}
$$

where

$$
\begin{gathered}
c_{1}=\gamma_{1}+k_{4} \bar{y}_{3}, \quad c_{2}=\frac{2 k_{1} k_{3} \bar{y}_{2}}{\delta^{2}}, \\
c_{3}=k_{4} \bar{y}_{1}, \quad c_{4}=\frac{k_{1} k_{3}}{\delta^{2}}, \quad c_{5}=k_{4} \bar{y}_{3} .
\end{gathered}
$$

It is seen that notations in Section 2 here are

$$
\begin{gathered}
f_{2}=f_{3}=f_{6}=g_{1}=g_{4}=g_{5}=g_{6}=h_{2}=h_{3}=h_{4}=0, \\
f_{1}=-c_{1}, \quad f_{4}=-c_{2}, \quad f_{5}=-c_{3}, \\
g_{2}=k_{5}, \quad g_{3}=-\gamma_{2}, \\
h_{1}=-c_{5}, \quad h_{5}=-c_{6}, \quad h_{6}=-k_{6} .
\end{gathered}
$$

In the next few figures we illustrate numerically the validity of the criteria proved Section 5, that is, when the three time 
delays are different from zero. For our simulations according to [22], the numerical values of the model parameters are

$$
\begin{gathered}
k_{1}=0.3\left[\mathrm{~min}^{-1}\right], \quad k_{2}=k_{3}=1\left[\mathrm{~min}^{-1}\right], \\
k_{4}=0.3\left[\mathrm{~min}^{-1}\right], \quad k_{5}=0.5\left[\mathrm{~min}^{-1}\right], \\
k_{6} \in[0.05,0.3], \\
\gamma_{1}=0.1\left[\mathrm{~min}^{-1}\right], \quad \gamma_{2}=0.2\left[\mathrm{~min}^{-1}\right], \\
\tau_{1} \in[1,8], \quad \tau_{2} \in[12,35], \\
\tau_{3} \in[10,300], \quad l=0.1 .
\end{gathered}
$$

In order to compare the predictions with numerical results, the governing equations of the model (40) were solved numerically using MATLAB [26]. In Figure 1(a), the stable solutions for the concentration of mRNA $\left(y_{1}\right)$, the concentration of protein $\left(y_{2}\right)$, and the concentration of miRNA $\left(y_{3}\right)$ are shown for absence of time delay (i.e., $\tau_{1}=\tau_{2}=\tau_{3}=$ 0 ). It is evident that after several physiological acceptance fluctuations, the solution of system (2) approaches a constant value (stable equilibrium state). In other words, the system possesses a stable equilibrium state which corresponds to a normal miRNA regulation process. This conclusion is in accordance with the criteria written in (7). In Figure 1(b), the unstable solutions of system (40) are shown. It is seen that for larger values of $\tau_{3}$, than bifurcation one, after Andronov-Hopf bifurcation the stable limit cycle (self oscillations) occurs and sustained oscillations take place. In other words, in this case the conditions of Theorem 9 are not satisfied and the steady state of system (40) is unstable.

\section{Conclusions}

In this paper we have considered a system of three equations with three discrete time delays. Under the assumption that an equilibrium exists, we have estimated the length of delays for which local asymptotic stability will be preserved. We have also derived criteria for which no change in stability will occur.

If system (2) starts with a stable equilibrium, which for some delay(s) becomes unstable, it will likely destabilize by means of an Andronov-Hopf bifurcation leading to small amplitude periodic solutions. Our investigation of such a behavior is devoted to the use of the bifurcation analysis. Particularly, a Hopf bifurcation theorem was employed. The basic view that the time delays $\zeta, \xi$, and $\eta$ are a key factor in the dynamical behavior of system (2) has been confirmed by analytical and numerical calculations.

\section{References}

[1] L. Chen and K. Aihara, "A model of periodic oscillation for genetic regulatory systems," IEEE Transactions on Circuits and Systems, vol. 49, no. 10, pp. 1429-1436, 2002.

[2] N. A. M. Monk, "Oscillatory expression of Hes1, p53, and NF$\kappa \mathrm{B}$ driven by transcriptional time delays," Current Biology, vol. 13, no. 16, pp. 1409-1413, 2003.
[3] C. Salazar and T. Höfer, "Kinetic models of phosphorylation cycles: a systematic approach using the rapid-equilibrium approximation for protein-protein interactions," BioSystems, vol. 83, no. 2-3, pp. 195-206, 2006.

[4] P. Smolen, D. A. Baxter, and J. H. Byrne, "Frequency selectivity, multistability, and oscillations emerge from models of genetic regulatory systems," American Journal of Physiology, vol. 274, no. 2, pp. C531-C542, 1998.

[5] D. M. Wolf and F. H. Eeckman, "On the relationship between genomic regulatory element organization and gene regulatory dynamics," Journal of Theoretical Biology, vol. 195, no. 2, pp. 167186, 1998.

[6] E. Almodaresi and M. Bozorg, "Stability crossing surfaces for linear time-delay systems with three delays," International Journal of Control, vol. 82, no. 12, pp. 2304-2310, 2009.

[7] S. Guo, L. Huang, and L. Wang, "Linear stability and Hopf bifurcation in a two-neuron network with three delays," International Journal of Bifurcation and Chaos in Applied Sciences and Engineering, vol. 14, no. 8, pp. 2799-2810, 2004.

[8] J. Llibre and A.-A. Tarţa, "Periodic solutions of delay equations with three delays via bi-Hamiltonian systems," Nonlinear Analysis, vol. 64, no. 11, pp. 2433-2441, 2006.

[9] S. Nikolov, "Stability and bifurcation behaviour of genetic regulatory systems with two delays," Comptes rendus de l'Academie bulgare des Sciences, vol. 61, no. 5, pp. 585-594, 2008.

[10] S. Nikolov, X. Lai, U. W. Liebal, O. Wolkenhauer, and J. Vera, "Integration of sensitivity and bifurcation analysis to detect critical processes in a model combining signalling and cell population dynamics," International Journal of Systems Science, vol. 41, no. 1, pp. 81-105, 2010.

[11] R. Ouifki and G. Witten, "Stability analysis of a model for HIV infection with RTI and three intracellular delays," BioSystems, vol. 95, no. 1, pp. 1-6, 2009.

[12] R. Sipahi and I. I. Delice, "Stability of inventory dynamics in supply chains with three delays," International Journal of Production Economics, vol. 123, no. 1, pp. 107-117, 2010.

[13] J. Wei and S. Ruan, "Stability and bifurcation in a neural network model with two delays," Physica D, vol. 130, no. 3-4, pp. 255-272, 1999.

[14] E. Hopf, "Abzweiging einer periodischen losung eines differential systems," Berichte der Mathematisch-Physikalischen Klasse der Sächsischen Akademie der Wissenschaften zu Leipzig, vol. 99, pp. 1-22, 1942.

[15] J. K. Hale and S. M. Verduyn Lunel, Introduction to FunctionalDifferential Equations, vol. 99 of Applied Mathematical Sciences, Springer, New York, NY, USA, 1993.

[16] M. Adimy, F. Crauste, and S. Ruan, "Periodic oscillations in leukopoiesis models with two delays," Journal of Theoretical Biology, vol. 242, no. 2, pp. 288-299, 2006.

[17] X. Li, S. Ruan, and J. Wei, "Stability and bifurcation in delaydifferential equations with two delays," Journal of Mathematical Analysis and Applications, vol. 236, no. 2, pp. 254-280, 1999.

[18] S. Ruan and J. Wei, "On the zeros of transcendental functions with applications to stability of delay differential equations with two delays," Dynamics of Continuous, Discrete \& Impulsive Systems, vol. 10, no. 6, pp. 863-874, 2003.

[19] Q. J. A. Khan and D. Greenhalgh, "Hopf bifurcation in epidemic models with a time delay in vaccination," IMA Journal of Mathemathics Applied in Medicine and Biology, vol. 16, no. 2, pp. 113$142,1999$. 
[20] R. May, Stability and Complexity in Model Ecosystems, Princeton University Press, 1973.

[21] J. E. Marsden and M. McCracken, The Hopf Bifurcation and Its Applications, Springer, New York, NY, USA, 1976.

[22] S. Nikolov, J. Vera, M. Nenov, and O. Wolkenhauer, "Dynamics of a miRNA model with two delays," Biotechnology \& Biotechnological Equipment, vol. 26, no. 5, pp. 3315-3320, 2012.

[23] Z. R. Xie, H. T. Yang, W. C. Liu, and M. J. Hwang, "The role of microRNA in the delayed negative feedback regulation of gene expression," Biochemical and Biophysical Research Communications, vol. 358, no. 3, pp. 722-726, 2007.

[24] S. Zeiser, H. V. Liebscher, H. Tiedemann et al., "Number of active transcription factor binding sites is essential for the Hes7 oscillator," Theoretical biology \& medical modelling, vol. 3, p. 11, 2006.

[25] G. A. Korn and T. M. Korn, Mathematical Handbook for Scientists and Engineers, McGraw-Hill, New York, NY, USA, 1968.

[26] "Matlab," The MathWorks, Natick, Mass, USA, 2010, http:// www.mathworks.com. 


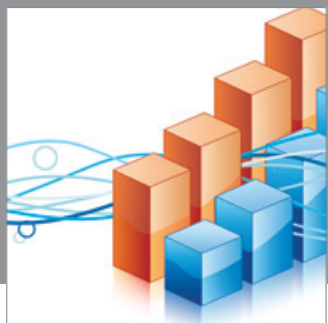

Advances in

Operations Research

mansans

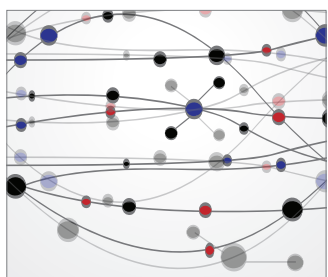

The Scientific World Journal
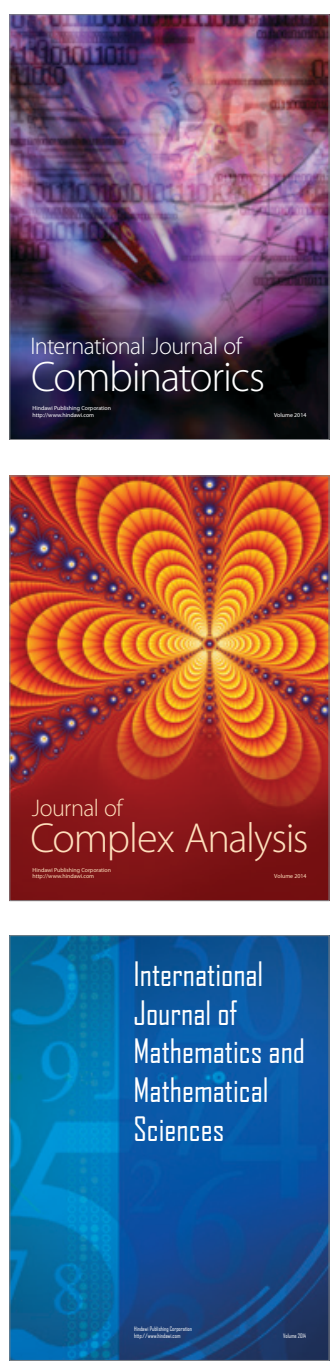
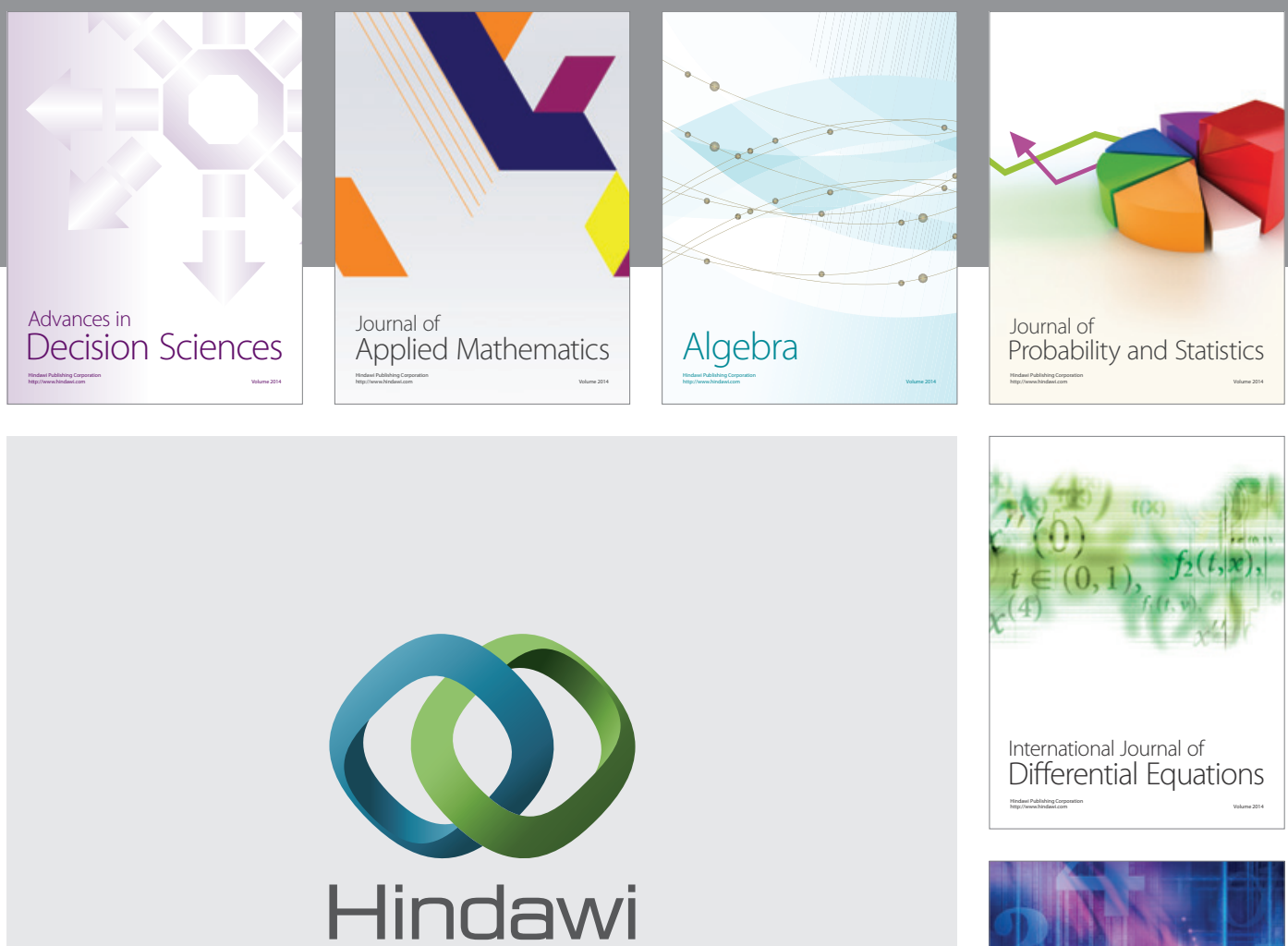

Submit your manuscripts at http://www.hindawi.com
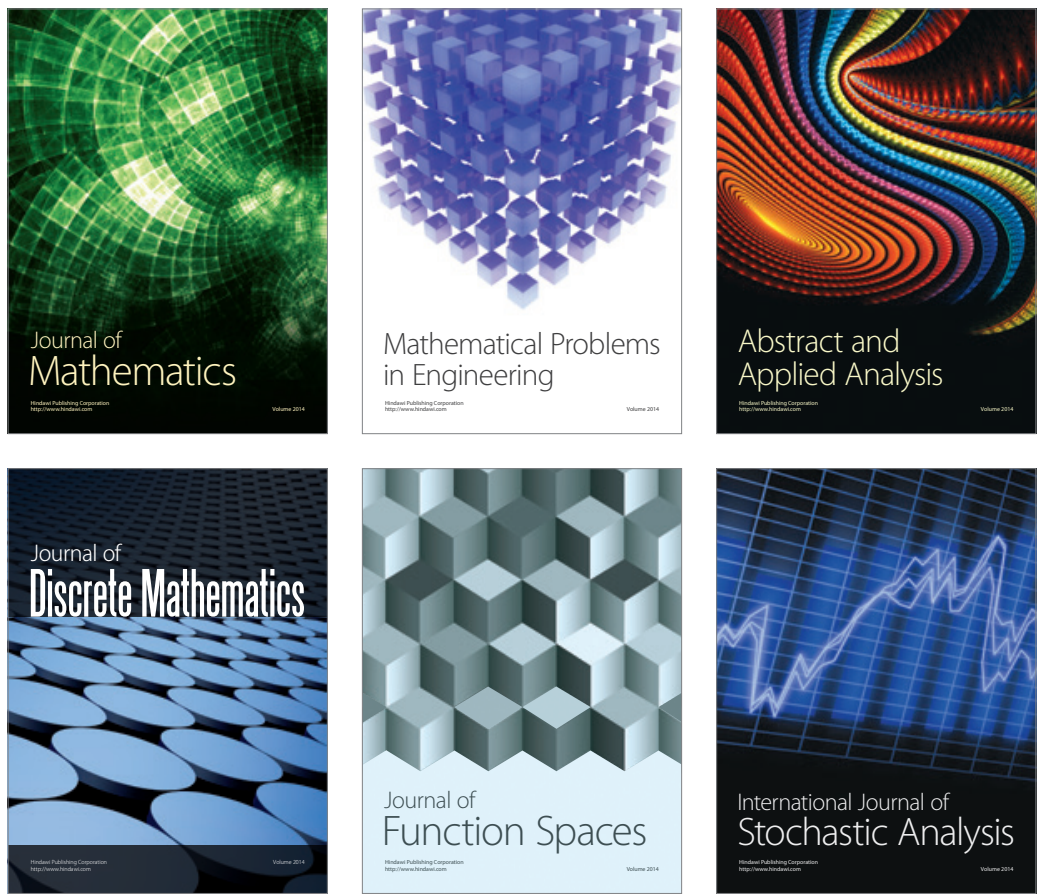

Journal of

Function Spaces

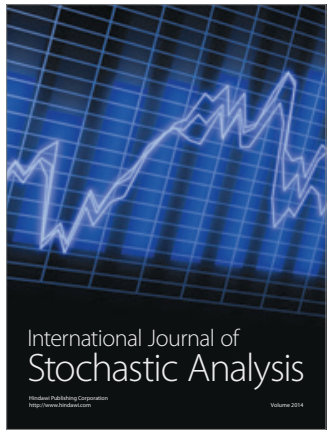

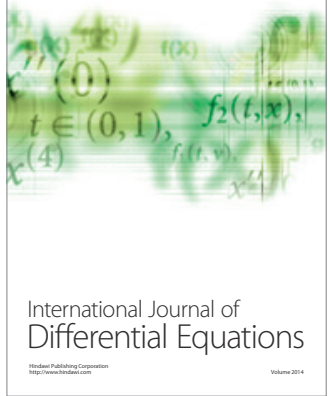
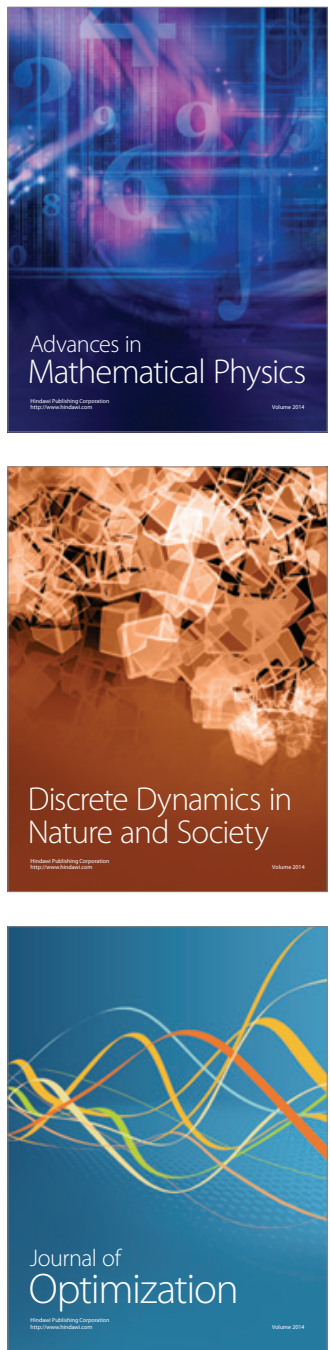